\title{
Australia en el Pacífico
}

\begin{abstract}
W. MACMAHON BALL, ha escrito $y$ ha efectuado comentarios radiales sobre aspectos relativos a la política exterior de Asia y Australia. Fue contralor de radiodifusión internacional, asesor de la Delegación de Australia a la Conferencia de San Francisco en 1945 , representante político de su país en las Indias Orientales Holandesas, miembro del Consejo Aliado para el Japón en representación de la Comunidad Británica de Naciones, y Ministro de su país en 1946 y 1947. Desde 1949 hasta 1968 fue profesor de ciencia política en la. Universidad de Melbourne. Publicó Japan Enemy or Ally? en 1949, y' Nationalism and Comunism in East Asia, 1956.
\end{abstract}

Los intereses nacionales básicos de Australia no tienen nada de extraño. Queremos seguridad física contra un ataque militar. Queremos mantener y elevar nuestro bienestar material, promover el crecimiento económico y la prosperidad social. Queremos preservar nuestro propio estilo de vida radicado en las tradiciones europeas $y$ predominantemente británicas, y limitar el influjo de gentes de diferente tradición o raza a pequeñas minorías escogidas que no engendren fricciones sociales o comunales.

Nuestras circunstancias son inusuales. Somos una pequeña nación de 13 millones de personas en un país de casi el mismo tamaño que los Estados Unidos. Vivimos en una isla frente a las costas de Asia, que alberga a más de la mitad de la población mundial. Los asiáticos son muchos, nosotros somos pocos. Salvo los japoneses, la mayoría de los asiáticos son pobres, mientras que nosotros somos ricos. A menudo ellos están superpoblados y apiñados, nosotros no. Son muy diferentes de nosotros en raza, lengua y religión.

Hasta la Segunda Guerra Mundial no parecía haber ninguna razón que nos obligara a comprometernos con Asia. En nuestras escuelas y universidades, la enseñanza de idiomas e historia, y aun de la geografía, estaba centrada en Europa Occidental y las Islas Británicas. Todo el Este y Sudeste asiático, salvo Japón y Tailandia, estaban controlados por potencias occidentales. China no era independiente más que de nombre, porque los "tratados desiguales" la privaban de los derechos básicos de un estado soberano. Nuestras relaciones oficiales con nuestros vecinos eran relaciones con sus sobera- 
nos británicos, holandeses, franceses o norteamericanos. Pero el nacionalismo ha transformado desde entonces el clima político de Asia, de modo que para Australia, Asia es no sólo un norte cercano en vez de un lejano oriente, sino también un nuevo norte.

Donde quiera que la gente llega a sentir que pertenece a una nación, y no sólo a una familia, tribu, clase o comunidad religiosa, comienzan a desear fervientemente establecer límites seguros alrededor de su territorio, tener su propio gobierno con un poder supremo dentro de esos límites, sin ninguna clase de subordinación a ningún gobierno extranjero. A partir de aquí, la nación tiende a convertirse en una nación-estado independiente.

En Asia durante este siglo, el nacionalismo ha constituido una revolución fundamental contra la dominación de los europeos. Los portugueses llegaron al sudeste de Asia en el siglo xvi, así como los españoles a las Filipinas, mientras en el siglo xvir llegaron al sur y al sudeste de Asia los holandeses; los británicos y los franceses lo hicieron en los siglos xvir y xIx. De aquí que algunos asiáticos hayan estado sometidos a la soberanía de europeos durante más o menos 400 años, y otros durante menos de 100.

El nacionalismo político ha sido, por lo general, fuertemente sostenido por una rebelión económica contra la explotación ejercida por los extranjeros. El nacionalismo económico en Asia no es sólo antioccidental. En algunos perfodos y lugares, ha sido antijaponés. En Birmania, no sólo ha sido antibritánico, sino también antiindio. En la mayoría de los países del sudeste de Asia, ha sido antichino, ya que la población indígena se ha resentido por el éxito comercial y el mejor nivel de vida que han logrado las minorías chinas. Es verdad que la administración europea acarreó generalmente un crecimiento económico en los territorios coloniales. Los europeos extrajeron y exportaron minerales, cultivaron y exportaron especias, té, café o cocos. En algunas áreas introdujeron y cultivaron el caucho. Pero el crecimiento económico no trajo necesariamente aparejado un incremento del bienestar social. De hecho, en algunos lugares y períodos, la reorganización de las economías asiáticas en pro de los intereses de los poderes coloniales acarreó un empobrecimiento material y espiritual de las poblaciones asiáticas. Aun cuando el desarrollo económico les trajo algún beneficio real, los intereses de los poderes coloniales eran siempre desmedidamente predominantes.

En varios países asiáticos, estos resentimientos políticos y económicos fueron ieforzados por resentimientos basados en la raza o el color. Es un hecho histórico triste y vergonzoso que muchos europeos en Asia dieron por sentado que el hombre blanco es inherentemente superior al de color. No hay duda de que la explicación de este error ridículo es que, durante las últimas centurias de lạ larga 
historia del mundo, algunas razas europeas alcanzaron un mayor adelanto que las asiáticas en cuanto a habilidades técnicas o indus. triales se refiere. En particular, construían cañones mejores y más grandes... Son fundamentalmente estas cosas las que les dieron una gloria evanescente en Asia.

De aquí que el moderno nacionalismo asiático busque tres liberaciones: la liberación de la dominación política, de la explotación económica y del rechazo racial. El nacionalismo en el Este y Sudeste asiático ha ganado un gran impetu a partir de la derrota de Rusia por el Japón en la guerra de 1904-1905. En este conflicto, el Japón ganó una serie de victorias destructoras en tierra y mar, que culminaron con la aniquilación de la flota rusa en la batalla de Tsushima, en 1905. Si una potencia asiática podía sobrepasar a una gran potencia europea en el arte y la ciencia de la guerra, tal vez otros países asiáticos podrían hacer lo mismo.

La Guerra del Pacífico y sus secuelas acarrearon victorias fundamentales para el nacionalismo asiático. Esta guerra mostró rápidamente que los poderes coloniales eran incapaces de hacer frente a su primera responsabilidad para con los pueblos que dependían de ellos, o sea protegerlos de todo ataque, invasión u ocupación. Los japoneses estaban prontos a dar a algunos de sus colaboradores en territorios ocupados un prestigio e influencia en el gobierno mayores que los que les eran proporcionados por sus amos europeos. No servía de nada que el Occidente denunciara a estos aliados como colaboracionistas o traidores. Después de todo, esos mismos pueblos se habían visto obligados a colaborar durante mucho más tiempo con los británicos, los franceses y los holandeses. X la mayoría de los asiáticos instruidos sentían que esa no era su guerra sino una guerra en la que sus gobemantes extranjeros los habían envuelto. Resulta irónico el hecho de que la mayoría de los líderes nacionales no comunistas hayan pasado parte de la guerra en las cárceles coloniales, mientras las autoridades europeas recibían gustosas la colaboración comunista contra el Japón.

Hacia el fin de la guerra, en 194.5, debió estar claro que la dominación europea en Asia había terminado. Los norteamericanos y los británicos se retiraron de esos territorios con alguna dignidad y realismo, pero los franceses y holandeses se retiraron luego de amargas luchas.

Sin embargo, desde entonces, los Estados Unidos, aliados con Australia han intervenido en dos guerras en el Este de Asia, la de Corea de 1950 a 1953, y la de Indochina durante los últimos 10 años. Esta guerra ha dividido profundamente a los australianos, pero el gobierno australiano, coalición conservadora formada por los partidos liberal y agrario, se ha identificado repetidamente con el punto de 
vista del gobierno norteamericano acerca de los problemas del oriente asiático. Estuvo de acuerdo con la prolongada insistencia del gobierno norteamericano acerca de la amenaza del comunismo chino, y con su diagnóstico de la guerra de Vietnam como una guerra de agresión por parte del estado de Vietnam del Norte contra el de Vietnam del Sur, en vez de una guerra civil. Nuestro gobierno ha endosado repetida y enfáticamente la estrategia norteamericana, en particular en cuanto a los bombardeos por aire y tierra contra Vietnam del Norte se refiere.

La simple razón por la cual el gobierno australiano se ha identificado tan estrechamente con la política norteamericana es que éste sabe que los Estados Unidos son la única nación occidental capaz de mantener o desplegar un poder militar efectivo en esta región, y que en consecuencia Australia confía en este poder para su seguridad.

Antes de considerar algunos de los problemas que se plantean para Australia a causa de la progresiva liberación de sus ataduras en Asia Continental, por parte de los Estados Unidos, y su política general de reducir sus compromisos en esa región, sería útil echar una mirada retrospectiva sobre la historia de la dependencia australiana.

Antes de 1914, pese a que Australia gozaba de un gobierno propio, todas las decisiones sobre política exterior eran tomadas por el gobiemo británico en nuestro nombre. $Y$ confiábamos en el poder marítimo y la diplomacia británicos para nuestra seguridad. El único poder asiático capaz de amenazarnos era el Japón, pero este país era aliado de Gran Bretaña desde 1902 (y hasta 1921). Después de la Primera Guerra Mundial, en la que las fuerzas expedicionarias australianas tomaron parte muy activa en Medio Oriente y en Europa, Australia logró un mayor status internacional a costa de 60 mil vidas. Tuvimos nuestra propia delegación a la Conferencia de Paz de 1919, y llegamos a ser miembro independiente de la Liga de las Naciones. Los compromisos que asumieran los británicos en sus tratados no obligaban más a Australia, aunque había gran discusión entre los expertos sobre si Australia podría permanecer, legalmente o de hecho, neutral en caso de que Gran Bretaña estuviera en guerra. Durante los veinte años del período de entreguerras, el gobierno británico informó generalmente al australiano acerca de sus decisiones en lo referente a política internacional, pero los gobiernos australianos continuaron protestando porque Londres rara vez consultaba a Canberra antes de tomar una decisión, de modo que Australia no tenía posibiliclad de compartir el proceso de adopción de la misma, aunque pudiera tener importantes consecuencias para nosotros. $\mathrm{Y}$ sin embargo, aún en 1938, Mr. Robert Menzies, ya por entonces una figura destacada del Gobierno del Commonwealth 
- $\mathrm{Y}$ posteriormente Primer Ministro entre 1939 y 1941 y nuevamente de 1949 a 1966 - declaró que no veía cómo podría Australia tener una política exterior propia, y que la línea correcta consistía en tratar, discreta y privadamente, de influenciar la política exterior británica en los casos en que los intereses australianos estuvieran en juego. Cuando estalló la guerra en Europa, en setiembre de 1939, nuestra Armada y nuestra Fuerza Aérea estaban comandadas por oficiales británicos "secundados". Nuestro Departamento de Asuntos Extranjeros había sido separado de la cartera del Primer Ministro recién en 1935, y sólo tenía un puñado de funcionarios. No había ninguna misión diplomática australiana en el exterior; sólo un oficial de enlace en Londres y otro en Washington. De hecho, cuando el gobierno designó a Mr. R. G. Casey como nuestro primer delegado en Washington y en cualquier otro lugar, el Primer Ministro Menzies enfatizó públicamente que esto en ningún modo significaba un debilitamiento del Imperio Británico.

La dependencia australiana respecto de Gran Bretaña era casi completa. Pero como Gran Bretaña luchaba contra Alemania e Italia, en ese momento prácticamente sola, muchos australianos llegaron a sentir que si Japón se sumara a la guerra, Gran Bretaña no podría desplegar fuerzas efectivas para la defensa de Australia y Nueva Zelandia, a 12 mil millas de las Islas Británicas. Cuando el Partido Laborista derrotó a la coalición conservadora, en octubre de 1941, el nuevo Primer Ministro, el señor John Curtain, y su Gabinete comenzaron a angustiarse progresivamente acerca de las intenciones del Japón. Pero los ingleses en Londres o en Singapur creían en alguna medida ser inexpugnables y se burlaron de las ansiedades australianas. Una revisión confidencial británica, sobre la situación en el Lejano Oriente, hecha a fines de setiembre, señalaba diversos puntos en los que dicha situación había ido evolucionandó en contra de los intereses del Japón, y declaraba que en consecuencia era muy poco probable que el Japón entrara en la guerra, lo que habría representado una locura.

A fines de octubre, el Sr. Churchill telegrafió al Sr. Curtain su opinión de que el Japón no se arriesgaría a una guerra, a menos que o hasta que Rusia hubiera sido aplastada, cosa que era poco probable en vista de la fuerte resistencia que los rusos venían oponiendo.

Cuando Japón descargó su golpe en Pearl Harbour, las Filipinas y Malaya, en diciembre de 1941, los australianos abrigamos la esperanza de que los norteamericanos, nuestros poderosos nuevos aliados, juntamente con los ingleses y holandeses, se recobrarían pronto de esa primera embestida. Los japoneses demolieron rápidamente estas ilusiones. Hacia fines de febrero de 1942, ya se había 
transformado el sentimiento australiano. Singapur había caído el 15 de febrero. Los cañones pesados estaban apuntados para disparar sólo hacia el mar, no hacia el continente al norte, en donde los japoneses habian atacado. Darwin fue bombardeada cuatro días más tarde, aunque la severidad del ataque fue ocultada largo tiempo por la censura. El gobierno y el pueblo australianos creían que la invasión y la ocupación subsiguiente eran lo más probable, mientras el bombardeo y cañoneo de las costas eran casi seguros.

Ya en diciembre de 1941, el Sr. Gurtain había marcado un viraje histórico en las relaciones exteriores australianas, haciendo público su llamamiento a los Estados Unidos para que protegieran a Australia, puesto que Gran Bretaña no estaba ya en condiciones de hacerlo. "Sin inhibiciones de ningún tipo, libres de remordimientos en cuanto a nuestras vinculaciones tradicionales o de parentesco con el Reino Unido, quiero dejar en claro que Australia mira hacia los Estados Unidos. Conocemos los problemas del Reino Unido. Conocemos la amenaza constante de invasión. Conocemos los peligros de la dispersión de fuerzas. Sabemos, también, que Australia podría caer y Gran Bretaña aun podría resistir. En consecuencia estamos decididos a que Australia no caiga, y ejercitaremos todas nuestras energías en la conformación de un plan, con los Estados Unidos como piedra angular, que dé a nuestro país alguna confianza en poder resistir hasta que la marea de la batalla refluya en contra del enemigo".

Tal vez resulte difícil para gente cuyo país ha sido derruido por la guerra, cuyas ciudades se han derrumbado bajo repetidos asaltos aéreos, entender el sentimiento del pueblo australiano, el sentido de sacudimiento y de mieclo que experimentó en los meses que siguieron al ataque japonés hacia el sur. Es que, probablemente, un pueblo no compara su situación con la de otros, sino con su propio pasado. Hasta 194:l, la guerra para los australianos había significado una expedición, no una invasión. En las guerras anteriores en las que Australia había luchado, inclusive la primera guerra mundial, no había existido una amenaza directa contra el suelo australiano. El peligro consistía en lo que podría ocurrirle a Australia luego de la guerra, luego de una derrota británica. De alli que las acciones japonesas en tierra australiana, aunque causaron una mínima pérdida de vidas y bienes en comparación con lo que otros paises sufrieron en la guerra, provocaron una conmoción y un temor profundos. $Y$ en tiempo de nuestro mayor peligro, fueron los Estados Unidos, y no Inglaterra, los que nos brindaron una protección efectiva.

Este es el trasfondo histórico del hecho de que, desde la segunda guerra mundial, el objetivo dominante de nuestra política exte- 
rior haya sido lograr que Norteamérica garantice la seguridad australiana. Nuestra alianza con los Estados Unidos, pese a la importancia crítica que nuestro gobierno le ha atribuido, no ha estado libre de molestias, frustraciones y algunos peligros. Por cierto, las frustraciones de Australia comenzaron con la alianza en tiempos de guerra. El gobierno australiano tardó en darse cuenta de que, aun después de Pearl Harbour, para Washington la derrota de Hitler tenía prioridad sobre la de Japón, y el Atlántico era más importante que el Pacífico.

El gobierno australiano reclamó urgentemente un arreglo formal que asegurara que su voz sería escuchada y atendida en Washington. Requirió el rápido establecimiento de un cuerpo encargado de la dirección de la Guerra en el Pacífico, en el cual Australia tuviera plena categoría de miembro. El Gabinete y el Consejo Asesor de Guerra multipartito fueron unánimes en esta petición. Pero fue en vano. Hubo algunos esfuerzos para aplacarnos, particularmente el establecimiento de un Consejo de Guerra en el Pacífico, con plena participación de Australia, pero ningún arreglo organizacional podía ocultar las realidades del poder. Roosevelt, en consulta con Churchill, tomó las decisiones estratégicas en el Pacífico. Ambos mostraron deferencia para con Stalin, ya que creían depender de Rusia para ganar la guerra en Europa, y podían necesitar la ayuda rusa para derrotar al Japón. Los tres creyeron que era necesario vencer a Hitler primero.

Aparte de las decisiones propiamente militares adoptadas durarite la guerra, los Estados Unidos decidieron generalmente en consulta con sus principales aliados, los objetivos de la guerra en el Pacífico y el Oriente de Asia y la política de posguerra hacia Japón. Para su desesperación y enojo, el gobierno australiano se enteró primero de las decisiones de largo alcance tomadas en El Cairo en diciembre del 1943, en Yalta en febrero de 1945 y en Postdam en julio de ese año, a través de la radio y la prensa. Tampoco el gobierno australiano habia sido consultado en modo alguno sobre la crítica y delicada cuestión de las negociaciones con el gobierno japonés en la primera mitad del mes de agosto de 1945, que llevaron a la aceptación de la declaración de Postdam y al fin de la guerra.

Siempre resueltos a ser tratados como factor principal en el Pacífico, los gobernantes australianos exigieron que Australia, como estaba estipulado, tuviera una parte prominente en la ocupación del Japón. Gran Bretaña, la India y Nueva Zelandia aceptaron que un personero designado por el gobierno no australiano -el autor de este artículo- fuera su representante conjunto ante el Consejo Aliado de Tokio. La Fuerza de Ocupación del Commonwealth Británico tenía un comandante en jefe australiano y el Tribunal Militar 
Internacional para el Lejano Oriente, un juez australiano como Presidente. $\mathrm{Y}$ sin embargo ese status no daba poder a Australia. La ocupación fue norteamericana, pese a la fachada institucional. Gradualmente, todos los principales objetivos australianos en relación con la ocupación fueron abandonados bajo persuasión o presión norteamericana. Mirando retrospectivamente, las políticas de los Estados Unidos hacia el Japón pueden considerarse en muchos aspectos como más inteligentes que las primitivas políticas australianas. Pero el hecho liso y llano era que incluso un grupo mayoritario de países aliados no podía alterar las decisiones norteamericanas a las que se oponían. Australia aprendió mucho sobre los inconvenientes de depender tanto de un amigo grande y poderoso. Sin embargo, después de todo, había sido el Sr. Menzies quien había dicho que la búsqueda de una política exterior por parte de un país pequeño es una búsqueda de amigos.

La prueba más dura para la política australiana para con Japón vino en 1951. El gobierno había sostenido firmemente hasta entonces el punto de vista de que el tratado de paz debía limitar efectivamente el rearme japonés, pero Washington se había opuesto con idéntica firmeza a toda restricción. Esto puso al gobierno australiano en una situación doméstica penosa, porque parecía que la mayoría de los australianos, y ciertamente el partido laborista y los sindicatos, se oponían agriamente al rearme irrestricto de Japón. El gobierno trató de persuadir a la gente de que el peligro presente y real no era más el Japón, sino la Unión Soviética y el comunismo, incluyendo a China Comunista; que debería importarnos más la seguridad del Japón que la seguridad contra el Japón.

Los problemas del gobierno fueron resueltos en parte por la negociación del pacto ANZUS, entre Australia, Nueva Zelandia y los Estados Unidos, que le dio una inmensa satisfacción. Este pacto podía ser presentado como un compromiso, por parte de la nación más poderosa del mundo, para proteger a Australia a la vez frente a la amenaza comunista que el gobierno enfatizaba y a la posible resurrección del militarismo japonés que enfatizaban sus críticos. Aun hoy el gobierno australiano proclama que el pacto ANZUS es el ancla de la seguridad australiana.

El gobierno australiano ha sufrido a veces molestias cuando Washington y Londres han seguido diferentes políticas en el Pacífico Asiático. En dos oportunidades, la década de 1950, pareció que los Estados Unidos podían verse envueltos en una guerra con China debido a las tensiones del Kuomintang en Formosa con China Continental. En ambos casos, el gobierno australiano, discreta y quedamente, indicó que se inclinaba hacia la opinión británica de que ese conflicto debía ser cuidadosamente evitado. 
A principios de 1954, los Estados Unidos, alarmados por la amenaza de un colapso militar francés en Indochina, campaña para la cual estaba proporcionando las tres cuartas partes del financiamiento, preguntó a sus amigos y aliados si estarían dispuestos a sostener, de una forma u otra, una intervención militar para salvar a Dien Bien Phu. Pero los aliados de los Estados Unidos no estaban dispuestos a hacerlo, y el presidente Eisenhower no quería hacerlo solo. Luego siguieron la retirada francesa y los acuerdos de Ginebra. Los Estados Unidos no tomaron parte en ellos, y sintieron que se necesitaba una acción unida para evitar una mayor expansión comunista por el suideste de Asia.

El gobierno australiano se había mostrado hábil al suplementar el pacto ANZUS -que importaba la defensa de los territorios australianos y neozelandeses, así como sus islas, barcos y aviones- mediante el compromiso norteamericano de defender el área del sudeste de Asia en general. Insistía en que, si era necesario luchar nuevamente para defender Australia, sería mucho mejor hacerlo en los países vecinos que en el nuestro. Esto era una actitud comprensible, aunque difícilmente podíamos esperar que nuestros vecinos asiáticos la aceptaran con un entusiasmo desmedido.

De aquí el apresurado establecimiento de la SEATO (Organización del Tratado del Sudeste de Asia), que se puso en vigor cuando sus ocho signatarios - Australia, Francia, Nueva Zelandia, Pakistán, Tailandia, Filipinas, el Reino Unido y los Estados Unidos- ratificaron el pacto en febrero de 1955. Laos, Camboya y Vietnam del Sur eran señalados como estados "designados", de modo que si algunos de sus gobiemos pedía ayuda, se les pudiera acordar. De todos modos, el gobierno australiano se sintió muy contento de que en la SEATO, por primera.vez, los Estados Unidos hubieran asumido un compromiso público, si bien no muy preciso, con la seguridad del Sudeste de Asia. Esto facilitaba que en los años siguientes el gobierno australiano proclamara su política de "defensa adelantada" y estacionara fuerzas militares australianas en el área.

Se ha vuelto cada vez más claro que la SEATO no tiene futuro, y por cierto apenas tiene pasado. Desde el principio tuvo debilidades de inválido. Ni la India, ni Birmania, ni Indonesia, ni Malaya, ni Laos, ni Camboya tenían interés en tomar parte en ella. Esto significaba que sólo incluía la quinta parte de la población y la tercera parte del territorio que debía proteger. Los Estados Unidos agregaron una "interpretación" del tratado, según la cual su propia obligación se limitaba a resistir una agresión comunista. Durante algún tiempo, Francia se había mostrado despectiva y ajena, cuando no abiertamente hostil al mismo. Los intereses británicos en el área nunca fueron muy fuertes, y tal vez se desvanecieron después 
de su retirada del Este de Suez. Paquistán ha anunciado ahora su retiro de la SEATO. Resulta evidente desde hace tiempo que cada miembro tiende a interpretar el tratado y sus provisiones de acuerdo con su propio juicio o interés. De aquí que la SEATO sea casi, si no totalmente, inútil para el sistema de seguridad en la región. Sin embargo, ha sido invocada a veces tanto por el gobierno norteamericano como por el australiano con el fin de justificar su intervención en Vietnam. Puede haber enfatizado así una imagen asiática de Australia como cliente y aliada militar de los Estados Unidos. A diferencia de nuestro gobierno, los de Gran Bretaña y Francia insistieron en que la SEATO no implicaba obligación alguna de sostener al gobierno de Vietnam del Sur.

Desde que nuestro gobierno comprometió nuestras fuerzas armadas en Vietnam en 1965, se ha visto a menudo aparentemente en una situación embarazosa. Desde entonces, el Gabinete, algunos de cuyos ministros han visitado Sudvietnam varias veces, ha continuado asegurando al pueblo australiano de que, pese a algunas decepciones, los norteamericanos y sus aliados estan efectivamente ganando la guerra -en el plano militar, en el programa de pacificación, y en la construcción de los cimientos de la democracia. -Aun ahora, -noviembre de 1972- el Primer Ministro, Sr. McMahon, declara públicamente que la reciente retirada de casi todas las fuerzas militares australianas de Vietnam es consecuencia de que los objetivos norteamericanos, y por consiguiente nuestros objetivos han sido logrados.

A través de toda esta guerra ha sido evidente a menudo que el gobierno norteamericano, como en el pasado, no ha consultado al australiano antes de tomar decisiones militares o políticas de gran envergadura. En 1968, dos prominentes Ministros del Gabinete, los de Defensa y Relaciones Exteriores, estaban explicando cuán importante era continuar con el bombardeo de Norvietnam, apenas un día o dos antes de que el Presidente Johnson anunciara la suspensión indefinida del mismo. El año pasado, el Primer Ministro condenaba al líder de la oposición por visitar Pekín siendo que el gobierno chino era un enemigo tan perverso y agresivo. Esto fue sólo un día o dos antes de que el gobierno se enterara, casi en el momento de despegar, que el presidente Nixon iba a visitar China.

Después de setenta años de dependencia de un gran poder -Gran Bretaña hasta 1941, y los Estados Unidos desde entonces- parece haber llegado el momento oportuno para que los australianos reflexionen sobre algunos rasgos de esta situación de dependencia, tal como la hemos conocido.

La amistad de un amigo poderoso es una cosa espléndida, pero no constituye una seguridad de que el amigo podrá dar una ayuda 
efectiva cuando más necesaria sea. Durante la Segunda Guerra Mundial, no hay duda de que el pueblo y el gobierno de Gran Bretaña querían hacer todo lo posible por defender Australia. Y sin embargo, para ellos era simplemente imposible hacerlo. La primera obligación del gobierno británico era para con el pueblo de las Islas Británicas; había que protegerlo de los bombardeos, la invasión o la ocupación, y mantener abiertas las rutas para importar alimentos y municiones. Era inevitable que la seguridad de Australia ocupara un lugar subordinado en la estrategia general británica.

Es adecuado que los australianos reflexionen sobre el hecho de que actualmente el Sudeste de Asia y el Sudeste del Pacífico pueden tener una prioridad bastante baja en la estrategia global de los Estados Unidos. En cierto sentido, Australia tuvo simplemente suerte al poder contar con la pronta y efectiva protección de los Estados Unidos durante la Guerra del Pacífico. Al atacar Pearl Harbour $\dot{y}$ las Filipinas, así como las fuerzas británicas y australianas en Malaya, Japón introdujo a los Estados Unidos en la guerra. $Y$ las pérdidas que Ios Estados Unidos sufrieron en Pearl Harbour, junto con la barrera protectora de bases insulares que Japón había construido para proteger las islas japonesas de un ataque por el este y el oeste, hicieron de Australia una base indispensable para un ulterior ataque en dirección de norte a sur. Fundamentalmente, debido a numerosos cambios tecnológicos ocurridos en los últimos treinta años, es improbable que esta cambinación vuelva a darse. Pese a algunas bases de comunicación instaladas por los Estados Unidos en Australia, es improbable que Australia tenga una importancia similar en la futura estrategia norteamericana a la que ha tenido en el pasado.

Es comprensible que el gobierno australiano ponga aún en público un gran énfasis en el pacto ANZUS, pero sería penoso que no comprendiera que en sí mismo no ofrece más que una frágil seguridad. Aunque los recursos norteamericanos son inmensos, no son infinitos, y el gobierno norteamericano tiene ahora compromisos y tratados con 42 naciones. No hay nada en el texto del ANZUS ni en el de la SEATO que se acerque a un compromiso de ayuda militar automática. Y la Doctrina Nixon, así como las acciones de Nixon, han aclarado al mundo que el gobierno de los Estados Unidos está buscando una revaluación de sus compromisos pasados a la luz de los intereses nacionales actuales o futuros. No es atacar la moralidad ni la sinceridad norteamericana pensar que sus obligaciones para con Australia se encontrarán subordinadas a sus obligaciones primarias para con el pueblo de los Estados Unidos.

No parece probable, luego de la agonía de Indochina, que algún gobierno norteamericano esté dispuesto a comprometer fuerzas terres- 
tres en el continente asiático en un futuro previsible. $\mathrm{Y}$ parece en cambio probable que los Estados Unidos reduzcan su amplio rango de compromisos de ultramar. Esto se debe al desencanto, si una palabra tan suave es la adecuada, con respecto a Indochina, pero también a la amarga desilusión frente a los resultados logrados hasta ahora a través de la transferencia masiva de ayuda técnica y económica a un gran número de países. Esta ayuda no ha conseguido ni la gratitud de esos países ni su apoyo sostenido a las politicas norteamericanas. $Y$ en buena medida tampoco ha conseguido promover el crecimiento económico en los países receptores. Más aún, muchos norteamericanos han empezado a sentir que deberían asignar una mayor proporción de sus recursos a las urgentes necesidades domésticas.

Parece que a partir de 1972, Australia deberá adaptarse a un cambio aún más fundamental en sus circunstancias externas que el de 1942. Nos sacudió entonces el descubrir que debíamos volvernos de Gran Bretaña hacia los Estados Unidos en busca de protección. Pero, tan traumático como eso haya podido ser, era sólo un viraje en nuestra dependencia, no el fin de ella. Hoy no parece haber ningún amigo grande y poderoso en cuya fuerza podamos basar nuestra seguridad física. Por primera vez, Australia deberá bastarse sola.

Esto no significa que Australia no tenga relaciones con el mundo. Nuestro comercio con Japón se ha elevado, de modo que Japón es actualmente nuestro principal mercado exportador, con la casi certeza de crecer más aún. Para Japón, Australia es un mercado pequeño, y una fuente muy importante de materias primas, en especial minerales. El gran incremento de la economía japonesa, y de su comercio, han aumentado considerablemente su influencia y prestigio políticos. El Japón desempeña, casi seguramente, un gran papel en la modelación del clima económico y político en esta parte del mundo. Los japoneses no tienen nuchas posibilidades de convertirse nuevamente en un formiclable poder militar, ni es probable que sus fuerzas armadas deseen combatir fuera del Japón o de las aguas japonesas. Es difícil divisar ninguna amenaza militar directa contra Australia en los próximos veinte años (y de hecho algunos australianos no han visto ninguna desde el fin de la Guerra de] Pacífico). Esto no significa que nos olvidemos de la clefensa. Quiere decir simplemente que tenemos tiempo para trabajar en un programa de defensa que sirva a la política exterior australiana, en vez de la inglesa o norteamericana.

Como estamos comparativamente adelantados en habilidades técnicas y administrativas, y somos comparativamente ricos, deberíamos poder hacer contribuciones útiles al desarrollo económico de los 
países del sudeste de Asia. Puesto que el comercio es a menudo más importante que la ayuda, estaríamos interesados en iniciar esfuerzos internacionales para estabilizar los precios de las principales mercancías exportadas por estos países.

Debemos recordar siempre que la política exterior es una combinación de políticas militares, diplomáticas y económicas, diferentes pero inseparables. $\mathrm{Y}$ muy importante sería reconocer que uno de los peores rasgos de nuestra dependencia pasada ha sido nuestra indebida subordinación a nuestro poderosos aliados para la comprensión del mundo -en el sentido más amplio- fuera de Australia.

Nuestra política futura debe construirse sobre un estudio activo, experto y profundo de lo que ocurra en la zona del Pacífico Asiático, de modo que nuestra política permanezca en línea con los cambios en nuestro alrededor.

Australia pertenece a la vez al Oriente y al Occidente. Nuestros intereses nacionales básicos consisten en evitar una guerra entre ellos, y reducir el abismo entre la pobreza asiática y el poder de Australia. 